\title{
Role of Perioperative Echocardiography in the Diagnosis of Hypertrophic Cardiomyopathy: Decoding Special Problem in the Background of Commoners
}

\author{
${ }^{1}$ Banashree Mandal, ${ }^{2}$ Sandeep S Rana, ${ }^{3}$ Ganesh K Munirathinam, ${ }^{4}$ Gurpinder S Ghotra
}

\begin{abstract}
We present a case posted for CABG with double valve replacement (DVR) for triple-vessel coronary artery disease (CAD), rheumatic severe mitral regurgitation, and severe aortic stenosis. However, preprocedure echocardiography in the operation theater confirmed the diagnosis of hypertrophic obstructive cardiomyopathy (HOCM) instead of valvular aortic stenosis, thereby altering the procedure performed to $C A B G$, mitral valve replacement (MVR), and myectomy.
\end{abstract}

Keywords: Coronary artery bypass graft, Hypertrophic cardiomyopathy, Perioperative echocardiography.

How to cite this article: Mandal B, Rana SS, Munirathinam GK, Ghotra SS. Role of Perioperative Echocardiography in the Diagnosis of Hypertrophic Cardiomyopathy: Decoding Special Problem in the Background of Commoners. J Perioper Echocardiogr 2017;5(1):27-30.

Source of support: Nil

Conflict of interest: None

\section{CASE REPORT}

A 72-year-old female patient came to our cardiovascular outpatient department with the chief complaint of chest pain of New York Heart Association grade II on and off for the past 5 months. Her past history revealed hematemesis 1 month back for which she had undergone sclerotherapy of the lower esophageal varices. She was a known hypertensive on oral medications. Her physical examination was normal except for the systolic murmur in the left third intercostal space radiating to the carotids and a systolic murmur in the left fifth intercostal region. The laboratory investigations including electrocardiogram and chest $X$-ray were within normal limits and

\footnotetext{
${ }^{1}$ Associate Professor, ${ }^{2}$ Professor, ${ }^{3,4}$ Fellow

1,3,4 Department of Anaesthesia and Intensive Care, Postgraduate Institute of Medical Education and Research, Chandigarh, India

${ }^{2}$ Department of Cardiothoracic and Vascular Surgery Postgraduate Institute of Medical Education and Research Chandigarh, India
}

Corresponding Author: Banashree Mandal, Associate Professor, Department of Anaesthesia and Intensive Care Postgraduate Institute of Medical Education and Research Chandigarh, India, Phone: +911722756500, e-mail: banashree mandal@yahoo.co.in the transthoracic echocardiogram by the cardiologist reported rheumatic severe aortic stenosis and moderate mitral regurgitation. The coronary angiogram showed significant triple vessel CAD. Hence, the patient was planned for DVR with CABG.

In the operating room, transthoracic echocardiogram done prior to anesthesia induction revealed mitral valve with mild stenosis and severe eccentric regurgitation. Two-dimensional imaging of apical five-chamber view showed asymmetrical basal septal hypertrophy $(2.1 \mathrm{~cm}$ in diastole), septal wall/posterior ratio 2.1/1.3 (1.6) (Figs 1A and $\mathrm{B})$. The aortic valve leaflets appeared normal with mild thickening of the edges and continuous wave Doppler across the aortic valve had a dagger-shaped envelope, peak and mean gradient of $71 / 33 \mathrm{~mm} \mathrm{Hg}$ respectively (Fig. 1C). The HOCM was suspected based on these findings. Once general anesthesia was induced, transesophageal echocardiography (TEE) was performed and the above findings were confirmed. The TEE showed normal aortic valve with minimal thickening of leaflets with the valve area of $1.8 \mathrm{~cm}^{2}$ in planimetry (Fig. 2A) and severe eccentric mitral regurgitation (Fig. 2B). In the deep transgastric view, color Doppler across the left ventricular outflow tract (LVOT) turbulence was noted in the LVOT away from the aortic valve (Video 1) and on applying pulse wave Doppler at different places of the LVOT, velocity increased away from aortic valve. Continuous Doppler showed a gradient of 106/50 mm $\mathrm{Hg}$ and dagger-shaped envelope confirming HOCM (Fig. 2C). After discussing with the cardiologist and the surgeon, the plan was changed to CABG, MVR, and myectomy of the basal interventricular septum. Keeping her recent history of sclerotherapy, initially we had planned for TEE only in midesophageal views but taking into account of her suspected HOCM, we progressed till transgastric view to visualize the LVOT thoroughly and did minimal manipulation in postbypass period. The patient had uneventful intensive care unit stay with no gastroesophageal bleed in any form.

At the end of surgery, patient came out of bypass with minimal inotropic support of norepinephrine $0.05 \mu \mathrm{g} / \mathrm{kg} / \mathrm{min}$. Postoperative TEE showed normally functioning bileaflet prosthetic mitral valve, good biventricular function (LV ejection fraction $=55 \%$ ) with 

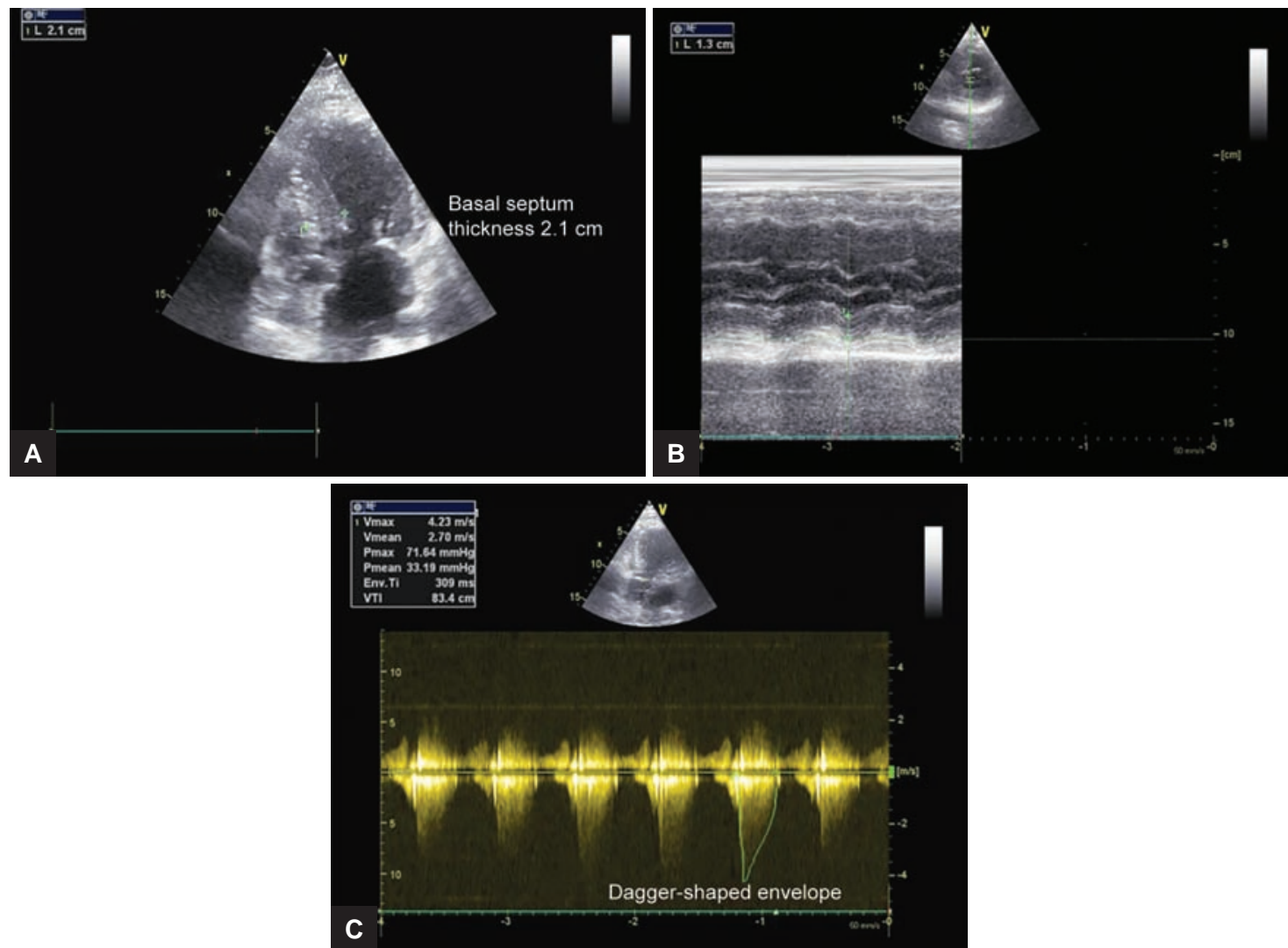

Figs 1A to C: Transthoracic echocardiographic. (A) Apical five-chamber view showing asymmetric basal septal hypertrophy $(2.1 \mathrm{~cm})$; (B) parasternal short-axis view showing posterior wall thickness $(1.3 \mathrm{~cm})$; and $(C)$ daggershaped continuous wave Doppler profile of the LVOT with a gradient of $71 / 33 \mathrm{~mm} \mathrm{Hg}$
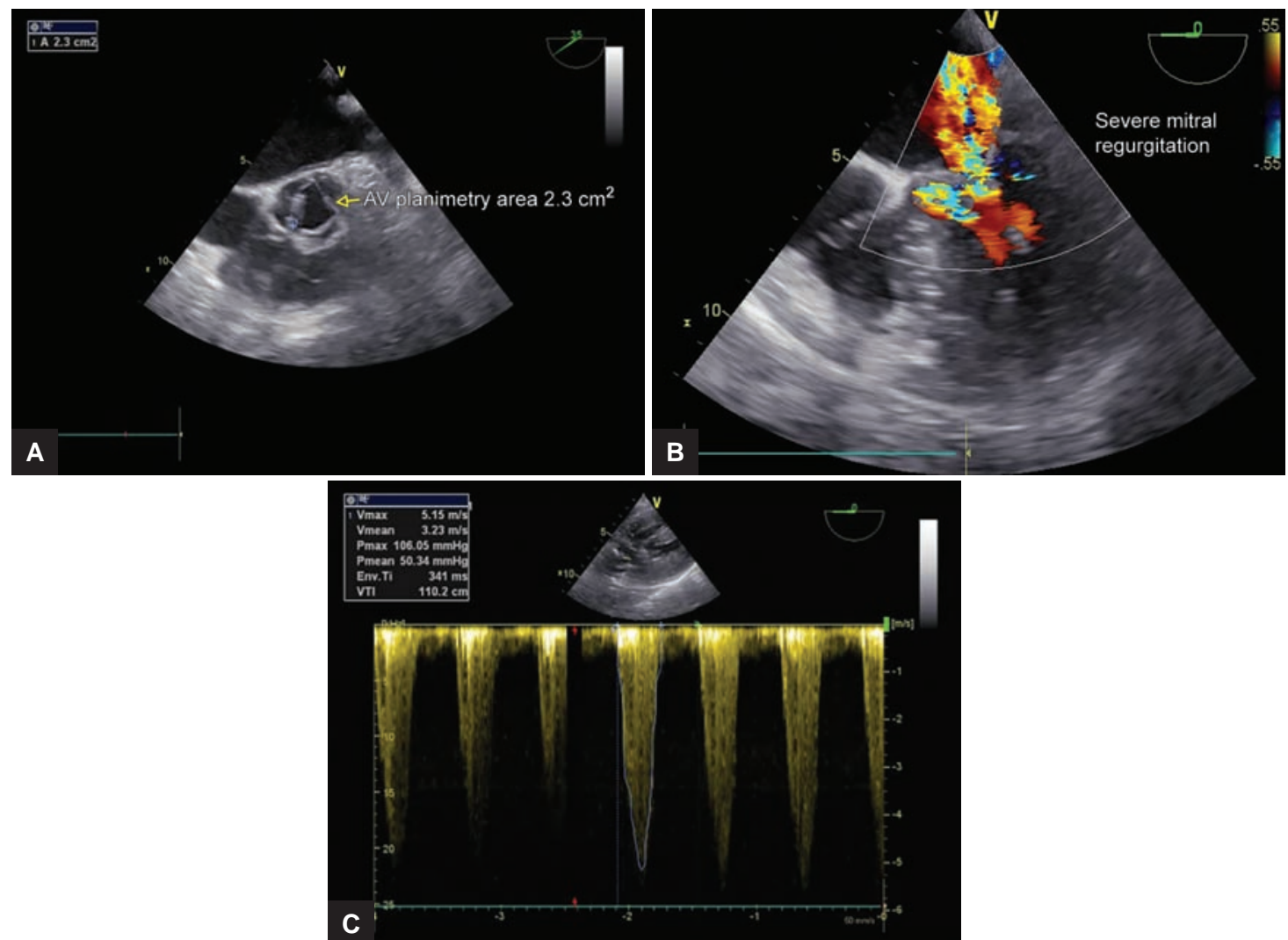

Figs 2A to C: Transthoracic echocardiographic. (A) Midesophageal aortic valve (AV) short-axis view showing planimetric measurement of AV area of $1.8 \mathrm{~cm}^{2}$; (B) midesophageal four-chamber view showing severe eccentric mitral regurgitation; and (C) deep transgastric long-axis view showing dagger-shaped continuous wave Doppler profile of the LVOT with a gradient of $106 / 50 \mathrm{~mm} \mathrm{Hg}$ 

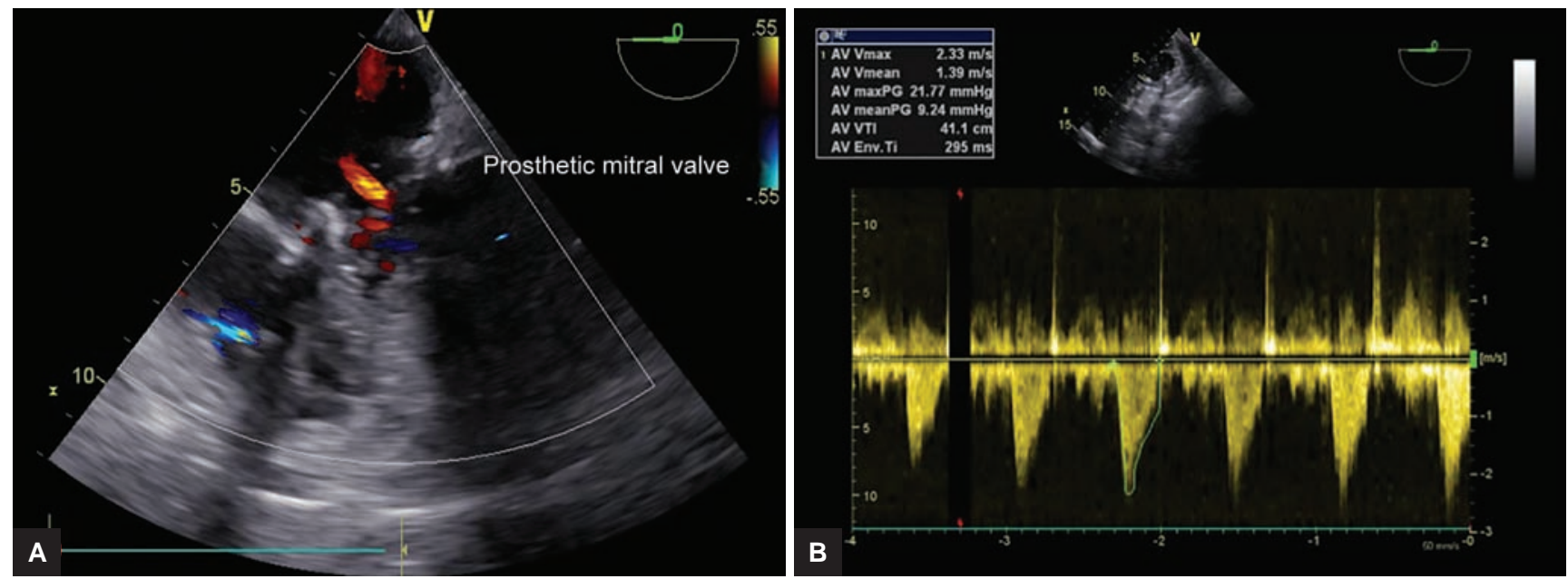

Figs 3A and B: Transthoracic echocardiographic. (A) Midesophageal four-chamber view showing functional prosthetic mitral valve; and (B) deep transgastric long-axis view showing the LVOT with a gradient of $21 / 9 \mathrm{~mm} \mathrm{Hg}$

no turbulence on color Doppler, and a gradient of 21/9 $\mathrm{mm} \mathrm{Hg}$ across the LVOT (Fig. 3, Video 2). In the intensive care unit, patient was ventilated for 6 hours and extubated. She got discharged to her ward on postoperative day 3 after an uneventful postoperative course. The histopathology report of the mitral valve tissue confirmed it to be rheumatic and septal tissue of HOCM.

\section{DISCUSSION}

Coronary artery bypass graft (CABG) surgery carries a higher morbidity and mortality when combined with other procedures like valve replacement. ${ }^{1}$ According to a study done in 1992 on American population, the incidence of significant CAD in patients with hypertrophic cardiomyopathy (HCM) was estimated to range from 7.6 to $19 \%$ in general but was as high as $24 \%$ in those over 45 years of age. ${ }^{2}$

In a more recent study done in 2015, the prevalence of obstructive coronary stenosis was approximately $16 \%$ in HCM patients without typical chest pain. ${ }^{3}$ Differentiating HCM from hypertensive heart disease is occasionally difficult. However, the study suggested that the significantly increased septum-to-lateral wall thickness ratios, and anyone of asymmetrical LV hypertrophy, hypertrophied right ventricular trabeculation in the interventricular septum, or abnormal regional LV bulging directs the diagnosis toward HOCM. ${ }^{3}$

Patients with rheumatic valvular heart disease presented with lower prevalence of CAD (4\%) when compared with nonrheumatic valvular heart disease (33.61\%) in a study of primary heart disease patients aged $\geq 40$ submitted to coronary arteriography. ${ }^{4}$ The prevalence of the disease was $6.95 \%$ among the patients aged $<60$ years, increasing to $31.8 \%$ among those aged $\geq 60$ years. ${ }^{4}$ In our part of the world, rheumatic heart disease is a common occurrence. The incidence of significant CAD was found to be nearly $12 \%$ in a study in Indian population with rheumatic heart disease. ${ }^{5}$ Valvular heart disease was sometimes overdiagnosed in a busy echocardiography lab. But keeping the rising risk of morbidity and mortality of combined procedure, we as perioperative echocardiographer do a thorough echocardiographic examination to avoid any unnecessary procedures. This case is a rare combination of rheumatic mitral valve disease with HOCM and CAD. The HOCM is diagnosed by echocardiographic features of asymmetric septal hypertrophy, increased septal-to-posterior wall thickness ratio, and presence of gradient in the LVOT. ${ }^{6}$ The striking echocardiographic features that we had in this case were asymmetric basal septal hypertrophy, color Doppler turbulence away from aortic valve, and dagger-shaped envelope of the continuous wave Doppler profile.

\section{CONCLUSION}

Immediate preoperative period as well as pre-CPB time provides an opportunity for the perioperative echocardiographer to confirm the preoperative findings and aim for a correct diagnosis by doing a comprehensive but systematic echocardiography by either transthoracic or transesophageal modality, which may help in avoiding unnecessary procedures.

\section{KEY POINTS}

- In elderly patients, the valvular abnormality that presents with significant CAD could be because of similar pathophysiology (i.e., atherosclerotic, sclerodegenerative) rather than rheumatic.

- Aortic stenosis in a setting of CAD should be thoroughly evaluated (aortic/subaortic).

- Perioperative echocardiography should be meticulously done as it helps in making major changes in surgical decision-making. 


\section{REFERENCES}

1. Nashef SA, Roques F, Sharples LD, Nilsson J, Smith C, Goldstone AR, Lockowandt U. EuroSCORE II. Eur J Cardiothorac Surg 2012 Apr;41(4):734-744.

2. Lazzeroni E, Rolli A, Aurier E, Botti G. Clinical significance of coronary artery disease in hypertrophic cardiomyopathy. Am J Cardiol 1992 Aug;70(4):499-501.

3. Okayama S, Soeda T, Kawakami R, Takami Y, Somekawa S, Ueda T. Evaluation of coronary artery disease and cardiac morphology and function in patients with hypertrophic cardiomyopathy, using cardiac computed tomography. Heart Vessels 2015 Jan;30(1):28-35.
4. Kruczan DD, Silva NA, Pereira BB, Romão VA, Correa Filho WB, Morales FE. Coronary artery disease in patients with rheumatic and non-rheumatic valvular heart disease treated at a public hospital in Rio de Janeiro. Arq Bras Cardiol 2008 Mar;90(3):197-203.

5. Jose VJ, Gupta SN, Joseph G, Chandy ST, George OK, Pati PK, John B, George P. Prevalence of coronary artery disease in patients with rheumatic heart disease in the current era. Indian Heart J 2004 Mar-Apr;56(2):129-131.

6. Shah, PM. Hypertrophic obstructive cardiomyopathy. In: Rapaport E, editor. Cardiology update. NewYork: Elsevier Biomedical; 1983. p. 175-191. 